\title{
Charlotte Murgier, Éthiques en dialogue, Aristote lecteur de Platon
}

Juliette Lemaire

\section{OpenEdition}

1 Journals

Édition électronique

URL : https://journals.openedition.org/philosant/698

DOI : 10.4000/philosant.698

ISSN : 2648-2789

Éditeur

Éditions Vrin

\section{Édition imprimée}

Date de publication : 1 novembre 2016

Pagination : 221-223

ISBN : 978-2-7574-1472-9

ISSN : 1634-4561

\section{Référence électronique}

Juliette Lemaire, "Charlotte Murgier, Éthiques en dialogue, Aristote lecteur de Platon », Philosophie antique [En ligne], 16 | 2016, mis en ligne le 01 novembre 2018, consulté le 07 décembre 2022. URL http://journals.openedition.org/philosant/698 ; DOI : https://doi.org/10.4000/philosant.698

\section{(c) $\oplus \Theta \Theta$}

Creative Commons - Attribution - Pas d'Utilisation Commerciale - Pas de Modification 4.0 International - CC BY-NC-ND 4.0

https://creativecommons.org/licenses/by-nc-nd/4.0/ 
l'ouvrage synthétique de $\mathrm{MB}$ dresse une remarquable cartographie du platonisme antique qui a le mérite de montrer comment se déploient des lignes de force, de continuité, ponctuées de bifurcations et de changements d'orientation. Cet essai, qui intègre le scepticisme académicien de plein droit dans l'héritage platonicien et qui souligne les passerelles entre l'époque impériale et le néoplatonisme, fait bouger les lignes d'opposition traditionnelles des commentateurs.

Alexandra MichaLewski CNRS, Centre Léon Robin

Charlotte Murgier, Éthiques en dialogue, Aristote lecteur de Platon, Paris, Vrin, 2014 (Textes et traditions), 352 p., ISBN : 978-2-7116-2532-1

Issu du remaniement d'une thèse de doctorat, cet ouvrage prend comme point de départ la critique d'un présupposé commun aux lectures néoplatonicienne et jaegerienne du rapport d'Aristote à Platon. D'un côté, les néoplatoniciens rendaient compatibles Aristote et Platon, tandis que Jaeger entendait montrer qu'Aristote avait progresssivement gagné son autonomie. Il n'en reste pas moins que ces deux démarches procèdent toutes deux à une reconstruction systématique de la philosophie platonicienne qu'elles s'efforcent ensuite de retrouver dans les textes aristotéliciens, que ce soit sous forme critique ou positive (introduction, p. 11). Charlotte Murgier (CM) entend contourner ce présupposé : même si le projet aristotélicien gagne à être lu en regard de la philosophie platonicienne, l'étalon que représentent les thèses métaphysiques de Platon pour mesurer la proximité ou la distance d'Aristote par rapport à Platon est moins opératoire en philosophie pratique, quand bien même Aristote critique l'Idée du Bien en Éthique à Nicomaque I, 4 (selon le découpage de Susemihl) et Éthique à Eudème I, 8. La critique aristotélicienne de l'Idée du Bien n'est pas le dernier mot du dialogue entre Aristote et Platon, mais bien plutôt son prologue. Il s'agit ainsi de « partir à la recherche d'une autre présence du platonisme dans l'éthique aristotélicienne » et d' «abandonner la flagrance du désaccord » (p. 12). Même si les mentions explicites de Platon sont rares dans l'EN, on y trouve des références précises, voire des reprises littérales de passages du Philèbe. Ainsi «le »Platon avec lequel Aristote discute est celui des dialogues celui que nous connaissons tous. Aristote est un lecteur de Platon, et non pas celui qui a purement et simplement rejetéle Bien. Ce parti pris conduit CM à éviter tout recours aux doctrines non écrites, et à prendre le platonisme comme une œuvre écrite, pour Aristote comme pour nous lecteurs de Platon, en faisant comme si Aristote ne connaissait que les Dialogues de Platon. Cela afin d'« échapper au cercle vicieux consistant à définir la philosophie platonicienne à partir d'une tradition... redevable au moins en partie de la source aristotélicienne », et afin d'échapper également « à la tentation d'opposer, voire d'imposer une interprétation du platonisme contre une autre, préférant le Platon reconstitué par nos soins à son portrait aristotélicien » (p. 13). Prenant au pied de la lettre le qualificatif de liseur que, selon la tradition, Platon lui-même aurait attribué à Aristote, $\mathrm{CM}$ entend considérer la construction des concepts éthiques aristotéliciens à partir de la lecture faite par Aristote des dialogues platoniciens, en mettant entre parenthèses la question socratique, c'est-à-dire la distinction problématique entre le Socrate « historique » et le Socrate de Platon. Montrer comment « l'héritage platonicien dans l'éthique aristotélicienne consiste ... moins dans la reprise, critique ou non, de thèses que dans la réappropriation de problèmes dialectiques issus des dialogues de Platon », tels sont l'interprétation et l'apport majeurs de cet ouvrage. Pour ce faire, les textes utilisés seront majoritairement tirés de l'EN et des Politiques, en excluant les 
fragments du Protreptique, le témoignage des Magna moralia et le livre VIII de l'EE. CM n'y relèvera pas de façon exhaustive toutes les références et allusions probables aux dialogues platoniciens, car son but n'est pas de recenser des thèses mais d'enquêter sur des textes, de révéler « que la reprise aristotélicienne ne se fait pas de loin, mais au plus près des textes de Platon » et de privilégier la problématicité au détriment de l'exhaustivité (p. 16). « La lecture attentive et rigoureuse des difficultés mises en scène par Platon » permet à Aristote de développer les questions éthiques, la philosophie platonicienne n'étant pas un endoxon parmi d'autres. Ainsi, les problèmes de Platon sont d'authentiques problèmes : la valeur du plaisir, la nature du savoir pratique et les conditions de possibilité de l'amitié « sont autant de problèmes platoniciens dont Aristote repart » (p. 17). La dialectique aristotélicienne, en travaillant sur les problèmes posés par Platon, va nous permettre de remonter aux principes de l'éthique, conformément à ce que déclarent les Topiques, I, 2, 101a25-b4 à propos de l'utilité de la dialectique. Cette lecture de l'éthique aristotélicienne avec des «lunettes » platoniciennes permettra ainsi de réévaluer la dette platonicienne d'Aristote, moins évidente en éthique, et d'entrer dans « la fabrique des concepts » aristotéliciens (p. 18). Les textes éthiques d'Aristote vont se révéler problématiques, plutôt qu'assertoriques « dès lors que s'y donne à lire la genèse logique, sinon chronologique, des solutions qu'ils proposent. En cherchant à comprendre le pourquoi d'un refus, on trouve aussi le comment d'une genèse, celle des concepts qui en constituent l'armature » (p. 18). On verra ainsi que la philosophie d'Aristote n'est pas un système statique de concepts : « À laisser ainsi le platonisme pour ainsi dire "ouvert", c'est l'aristotélisme qui s'en trouve à son tour vivifié » (p. 19).

Trois parties sont consacrées à cet Aristote lecteur, en dette à l'égard de Platon. La première, consacrée au Bien pratique, analyse comment s'opère le passage du bien platonicien au bien aristotélicien (chap. I, p. 23-67), puis examine comment se noue le rapport du bien et du plaisir dans le texte du Philèbe de Platon pour analyser la manière dont Aristote repart de ce nœud pour construire son propre concept du plaisir (chap. II, p. 69-120). Partant de la définition aristotélicienne du plaisir comme activité, la deuxième partie opère le passage de l'activité à l'action : d'abord, l'analyse des « plaisirs de la vie vertueuse », dans la contemplation, puis dans la vie éthique (chap.. III, p. 123-154), et ensuite, celle de « l'amitié ou [du] plaisir d'agir » (chap.. IV, p. 155-197). La troisième partie, «De l'action à sa perfection : autour de la phronesis » se concentre sur cette notion fondamentale de l'éthique aristotélicienne qu' est la phronesis, montrant d'abord sa genèse à partir du Ménon (chap.. V, p. 201-261), puis analyse comment le savoir pratique que constitue la phronesis acquiert une dimension politique (chap. VI, p. 263-294).

Aristote est lecteur de Platon, tout particulièrement en éthique ; le texte de Platon est une matrice pour les concepts aristotéliciens. Plus personne ne peut douter de ces deux propositions après la lecture de cet ouvrage. La manière dont CM démontre ces propositions doit être louée pour sa belle expression minutieuse, sa patience, son exhaustivité, sa clarté et son originalité dans l'appel à des philosophes bien plus tardifs qu'Aristote - Kant, Caillois, ... - pour éclairer certains concepts, comme celui du jeu par exemple. Son analyse des textes platoniciens est aussi pertinente que celle des textes aristotéliciens, et sa connaissance des débats des interprètes est sans défaut. La fabrique conceptuelle aristotélicienne est véritablement mise en évidence. Pour prendre un seul exemple, on voit précisément comment Aristote repart de la question du plaisir telle qu'elle est posée dans le Philèbe et quelle torsion il fait subir au concept platonicien du plaisir. Socrate établissait que la vision appartient aux plaisirs purs et à la vie heureuse. Mais Aristote, lui, au lieu de considérer les plaisirs purs comme une espèce au sein d'un 
genre, va dégager grâce à eux la formule générale du plaisir. « Le paradigme » n'est «plus une figure d'exception » mais « un modèle générique pour la compréhension » (p. 99). Ainsi, les analyses textuelles serrées de CM, aussi bien des textes platoniciens que des textes aristotéliciens, permettent de saisir l'élaboration conceptuelle d'Aristote, que ce soit sur la question du bien, du plaisir, de la philia ou de la phronesis et du savoir politique. Cependant, on regrette quil n'y ait pas un paragraphe ou deux plus explicites sur la méthode de l'éthique - même s'il est fait référence aux articles incontournables sur la question, une partie sur la méthode propre à l'éthique et son rapport à la dialectique aristotélicienne pourrait encore davantage mettre en évidence la spécificité d'Aristote par rapport à Platon. On trouve certes des remarques évoquant la méthode dialectique au gré des analyses des textes aristotéliciens. Par exemple à propos de la réfutation opérée par Aristote du plaisir conçu sur le modèle de la réplétion, CM nous dit que « l'étiologie qui est en même temps une psychologie de l'erreur », est faite par Aristote « dans un geste typique de la méthode dialectique $\gg($ p. 88$)$ et renvoie alors au passage capital de l'EN sur cette question (VII, 15, 1154a21-25). Mais on aimerait en savoir davantage.

Qu'Aristote soit le liseur de Platon, particulièrement en éthique, cela est maintenant avéré. Cependant, ce choix de prendre la lettre platonicienne (ou plutôt des lunettes) pour comprendre la genèse des concepts aristotéliciens pourrait être un frein à la compréhension de l'EN et de l'EE pour elles-mêmes. Ainsi, pourquoi, après l'analyse du bien, en venir au plaisir, tel qu'il est déterminé au livre VII mais surtout au livre X, et à la vie contemplative, avant la vie éthique, puis passer par l'amitié pour enfin en arriver à la phronesis ? Il semble parfois que le mouvement et la progression de l'EN sont oubliés du fait de l'attention donnée au texte platonicien - il est certes toujours délicat de parler de plan d'ouvrage dans le cas d'Aristote, mais on peut cependant déceler une certaine cohérence dans l'EN. Précisément parce que dès I 6, les deux dimensions de l'activité de l'âme rationnelle sont posées, comme l'énonce explicitement CM, pourquoi avoir traité du plaisir de la vertu éthique après le plaisir de la theoria ? Car dès qu'Aristote analyse la vertu éthique au livre II de l'EN, il thématise la dimension du plaisir liée à l'activité. La contemplation n'est pas la seule activité humaine qui donne du plaisir. Certes, Aristote en fait l'activité la plus plaisante parce que la plus parfaite. Mais il reste que l'EN s'adresse aussi aux politiques, que l'éthique est bordée par la politique et que la theoria n'est pas la chose du monde la mieux partagée. En outre, la mention du but de l'éthique, que ce soit dans l'EN, II, 2, 1003 b27 ou dans l'EE, I, 5, 1216b6-9 - à savoir devenir vertueux, et non pas seulement connaitre la définition de la vertu - n'est pas exploitée, alors même que se critallise dans ces quelques mots une véritable invention de la part d'Aristote, i.e. l'éthique. Certes, la valeur du plaisir, la nature du savoir pratique et les conditions de possibilité de l'amitié sont des problèmes platoniciens d'où Aristote repart. Mais ce ne sont pas les seuls concepts éthiques aristotéliciens fondamentaux. On regrette ainsi que le plan de ce livre ait été dicté par les problèmes hérités de Platon et qu'il n'y ait pas eu davantage de pages sur la vertu et les vertus éthiques, alors même que l'analyse du prakton de CM ou son analyse de l'argument de l'ergon pour définir le propre de l'homme dans l'EN et l'EE sont essentielles à la compréhension de l'éthique aristotélicienne.

Ces ultimes remarques, expression d'une déception, n'entament en rien l'excellence de ce livre que toute personne intéressée par l'éthique et sa naissance dans l'antiquité grecque se doit de lire. 